\title{
On the Average Case Performance of Some Greedy Approximation Algorithms For the Uncapacitated Facility Location Problem
}

\author{
Abraham D. Flaxman \\ Alan M. Frieze* \\ email abie@cmu.edu \\ email alan@random.math.cmu.edu \\ Juan Carlos Vera \\ email jvera@andrew.cmu.edu \\ Department of Mathematical Sciences \\ Carnegie Mellon University \\ Pittsburgh, PA, 15213, USA
}

May 4, 2006

\begin{abstract}
In combinatorial optimization, a popular approach to NP-hard problems is the design of approximation algorithms. These algorithms typically run in polynomial time and are guaranteed to produce a solution which is within a known multiplicative factor of optimal. Unfortunately, the known factor is often known to be large in pathological instances. Conventional wisdom holds that, in practice, approximation algorithms will produce solutions closer to optimal than their proven guarantees. In this paper, we use the rigorous-analysis-of-heuristics framework to investigate this conventional wisdom.

We analyze the performance of 3 related approximation algorithms for the uncapacitated facility location problem (from [Jain, Mahdian, Markakis, Saberi, Vazirani, 2003] and [Mahdian, Ye, Zhang, 2002]) when each is applied to an instances created by placing $n$ points uniformly at random in the unit square. We find that, with high probability, these 3 algorithms do not find asymptotically optimal solutions, and, also with high probability, a simple plane partitioning heuristic does find an asymptotically optimal solution.
\end{abstract}

*Supported in part by NSF Grant ccf0502793 


\section{Introduction}

Many optimization problems are NP-hard. This is an unfortunate fact of life. There are a variety of approaches to dealing with this problem. One approach is to find approximation algorithms with provably good worst-case performance guarantees. Another approach is to design heuristics which work well "on average". In this paper we will combine these two approaches and analyze an approximation algorithm in a probabilistic setting. The aim is to investigate the notion that such algorithms will "typically" do better than their worst-case guarantees. This type of analysis is differs from the more common approach in probabilistic analysis, where the algorithm is designed with the probability distribution of inputs in mind. It has been used in the study of approximation algorithms for MAX-CUT [9], the stacker crane problem [7], and bin packing [6].

In the uncapacitated facility location problem (UFLP) we are given a set of facilities $\mathcal{F}$ and a set of cities $\mathcal{C}$. For every facility $i \in \mathcal{F}$ there is a cost $f_{i}$ for opening that facility, and for every facility-city pair $(i, j) \in \mathcal{F} \times \mathcal{C}$ there is a cost $c_{i, j}$ for connecting facility $i$ to city $j$. There are no bounds on the number of cities that can be connected to a facility. Thus, if we open the set of facilities $F \subseteq \mathcal{F}$ then each city $j$ will connect to the open facility with cheapest connection cost, and the total cost will be

$$
c(F)=\sum_{i \in F} f_{i}+\sum_{j \in \mathcal{C}} \min _{i \in F} c_{i, j}
$$

The goal is to find a set of facilities $F$ that will minimize the total cost $c(F)$.

Unfortunately, the problem is NP-hard, as it contains set-cover as a special case. It has been the focus of a great deal of attention from many perspectives. In the 1980's, the Operations Research community focused on branch and bound algorithms for solving it, which led to some considerable success, see for example [13]. From that period, there is also some worstcase analysis of the performance of greedy heuristics [8] and a probabilistic analysis of the related $k$-median problem [1]. More recently, the Theoretical Computer Science community has placed a significant emphasis on finding approximation algorithms for NP-hard problems and one of its most notable successes has been in finding constant factor approximations for this problem when the connection costs obey the triangle inequality. The first algorithm to obtain a constant factor approximation was based on LP rounding [17] and subsequent approaches based on LP rounding improved the constant to $1+2 / e$ [5] and then to 1.58 [19]. Alternative approaches to approximating the solution are based on local search techniques [14], primal-dual schema [12] and combinations of these [4]. At the present time the best approximation guarantee that is obtainable in polynomial time is 1.52, due to Mahdian, Ye and Zhang [15]. This is a greedy augmentation algorithm, and in the present paper, we will focus our attention on it and on 2 related greedy algorithms [11].

It is likely that approximation algorithms will find solutions closer to optimal than their guarantees guarantee. How much closer? One way to provide some answer to this question 
is via an experimental study, which is exactly the approach of $[2,10]$ and is also considered in Section 7 of [11]. Another way, which we will follow in this paper, is to consider theoretically the result of applying the algorithms to an appropriate random instance. Since the constant factor approximation algorithms are only supposed to work on metric instances, we rule out one common random model, in which all distances are chosen independently and uniformly from $[0,1]$. Another random model we do not study comes from choosing all distances from a discrete distribution that takes only the values 1 and 2 . The random model we use will be geometric in nature, formed by placing points uniformly at random in the unit square. For additional reference on combinatorial optimization over instances derived from random points, see $[16,18,20]$. Although it is possible to design algorithms to take advantage of the special structure of these instances, that is not the focus of the current investigation. Instead of first choosing a distribution over instances and then designing an algorithm to work whp over this distribution, we begin by choosing the algorithms to study and then choose an interesting (but tractable) distribution of instances on which to run them.

\subsection{Random model}

We will study random instances formed by choosing $n$ points $\mathcal{X}=\left\{X_{1}, X_{2}, \ldots, X_{n}\right\}$ uniformly at random in the unit square $[0,1]^{2}$. We assume that each point represents a city and also the possible location of a facility. For simplicity we will use the $\ell_{\infty}$ distance between each facility-city pair as the connection cost (the techniques presented below would also work for the $\ell_{1}$ norm, but for the $\ell_{2}$ norm, additional effort would be needed to replace the results from Section 2).

Let $m$ be a positive integer satisfying $m=o\left((n / \log n)^{1 / 2}\right)$. Then let $\alpha=m^{-1}$ and define $\omega=m^{-1}(n / \log n)^{1 / 2}$, so that $\omega \rightarrow \infty$ with $n$.

We will give every facility the same opening cost,

$$
f=\frac{1}{6} \alpha^{3} n
$$

We have selected these values for later convenience in notation, and summarize it in the following table. It is really the facility cost $f$ that controls the structure of the optimal solution. As $f$ tends to $\infty$, the optimal solution will open 1 facility in the center of the square and connect everything to it. As $f$ tends to 0 , the optimal solution will open a facility at every city. Section 2 will show that the transition between these extreme behaviors is described by $f$ as parameterized above. For $f=\frac{1}{6} m^{-3} n$, the optimal solution will open about $m^{2}$ facilities. 


$$
\begin{aligned}
\omega \rightarrow \infty \quad m & =\omega^{-1} \sqrt{\frac{n}{\log n}} \quad \alpha=\omega \sqrt{\frac{\log n}{n}} \\
f & =\frac{1}{6} \omega^{3} \frac{(\log n)^{3 / 2}}{\sqrt{n}}
\end{aligned}
$$

We denote the $\ell_{\infty}$ distance between two points $X_{i}$ and $X_{j}$ by $d\left(X_{i}, X_{j}\right)$. All logarithms are base $e$.

We initially expected to prove that the algorithm of [15], which has worst-case approximation ratio 1.52 , was asymptotically optimal i.e. that $\mathbf{w h} \mathbf{p}^{1}$, as $n \rightarrow \infty$, the ratio of the cost of the solution found by the approximation algorithm and the optimum tends to 1 . Instead we give a proof of the following: Let OPT denote the value of a minimum cost solution. The algorithm of [15] is similar in spirit to the 2 algorithms given in [11], which have worst-case approximation ratios of at most 1.861 and 1.61. We denote these approximation algorithms by $H_{1}, H_{2}, H_{3}$, and recall their descriptions in detail in Section 2 . We let $Z_{i}$ denote the value of the solution found by $H_{i}$.

Theorem 1. There exists a positive constant $\epsilon>0$ such that for $i=1,2,3$, whp

$$
\frac{Z_{i}}{O P T} \geq 1+\epsilon
$$

On the other hand it is not difficult to describe a "trivial heuristic" which is asymptotically optimal and so it is disappointing that these sophisticated approximation algorithms are in fact beaten by triviality whp.

\subsection{Outline}

In the next section we describe the greedy approximation algorithms and the trivial heuristic in detail, and give a non-rigorous explanation of "what goes wrong" to prevent the approximation algorithms from finding an asymptotically optimal solution.

Since our non-rigorous explanation will rely heavily on the asymptotic optimality of the trivial heuristic, we prove that the heuristic is asymptotically optimal in Section 3. The proof has 2 parts. First we obtain an upper bound that holds whp on the value of the solution found by the heuristic. Since the heuristic is so simple, this only requires us to consider basic probabilistic arguments. Some of these recur frequently enough to merit little lemmas, which are stated and proved in Section 3.1. Then we obtain an asymptotically matching lower bound that holds whp on the value any solution. We do this by constructing a solution to the dual of the LP-relaxation which is feasible whp.

\footnotetext{
${ }^{1}$ A sequence of events $\mathcal{E}_{n}$ occurs with high probability $(\mathbf{w h} \mathbf{p})$, if $\lim _{n \rightarrow \infty} \operatorname{Pr}\left(\mathcal{E}_{n}\right)=1$
} 
The remainder of the paper proves Theorem 1. To do so, in Section 4.1, we state and prove some lemmas which show that the structure of any near optimal solution must take a certain form; it must choose facilities to open so that, for most open facilities, the region of the plane which is closer to that facility than any other is approximately a square of a certain size and is approximately centered on the facility. Lemma 5 from Section 4.1 is a quantitative version of this. Roughly, it says that if there are $\epsilon n$ facilities opened which violate these conditions then the solution will be a $1+\delta$ factor away from optimal.

To complete the proof of Theorem 1, in Section 4.2 we show that the approximation algorithms from Section 2 open too many facilities which do not meet the requirements for a close to optimal solution.

\section{Approximation Algorithms}

The approximation algorithms we consider are all similar. We first recall Algorithm 1 of [11] (which is most convenient for us in its restated form).

\section{Approximation Algorithm 1}

(a) The algorithm starts at time 0. Initially, each city is defined to be unconnected. The set of unconnected cities is denoted by $U$. All facilities are considered to be unopened and $\delta_{i}=0$ for $i \in C$, the set of cities.

(b) While $U \neq \emptyset$, increase the time and simultaneously for every city $i \in U$ increase the parameter $\delta_{i}$ at the same rate, until one of the following events occurs:

1. For some unconnected city $i$, and some open facility $j, \delta_{i}=d(i, j)$. In this case, connect city $i$ to facility $j$ and remove $j$ from $U$.

2. For some unopened facility $j, \sum_{i \in U} \max \left\{0, \delta_{i}-d(i, j)\right\}=f_{j}$. In this case open this facility and for every unconnected city with $\delta_{i} \geq d(i, j)$, connect $i$ to $j$ and remove it from $U$.

Now we recall Algorithm 2 of [11], which is very similar to Algorithm 1, but allows connected cities to contribute funds towards opening additional facilities.

\section{Approximation Algorithm 2}

(a) The algorithm starts at time 0. Initially, each city is defined to be unconnected. The set of unconnected cities is denoted by $U$. All facilities are considered to be unopened and $\delta_{i}=0$ for $i \in C$, the set of cities. We denote by $\pi$ the mapping from connected cities to open facilities. 
(b) While $U \neq \emptyset$, increase the time and simultaneously for every city $i \in U$ increase the parameter $\delta_{i}$ at the same rate, until one of the following events occurs:

1. For some unconnected city $i$, and some open facility $j, \delta_{i}=d(i, j)$. In this case, connect city $i$ to facility $j$ and remove $j$ from $U$.

2. For some unopened facility $j$, we have

$$
\sum_{i \in U} \max \left\{0, \delta_{i}-d(i, j)\right\}+\sum_{i \notin U} \max \left\{0, c_{i, j}-c_{i, \pi(i)}\right\}=f_{j}
$$

In this case open this facility and for every unconnected city with $\delta_{i} \geq d(i, j)$, connect $i$ to $j$ and remove it from $U$, and for every connected city with $c_{i, j}<c_{i, \pi(i)}$ change the facility to which $i$ connects from $\pi(i)$ to $j$.

Now, we recall Algorithm 3, which appears in [15] and currently has the best proven bound on worst-case approximation ratio.

\section{Approximation Algorithm 3}

(a) In the first phase, the algorithm scales up the opening costs of all facilities by a constant $\delta=1.504$, and uses Algorithm 2 to find a solution to the problem with these new costs.

(b) In the second phase, the algorithm considers the unmodified costs and performs a greedy augmentation to the solution found in phase 1 . Let $C$ denote the total connection cost in the phase 1 solution. For each unopened facility $j$, let $C_{j}$ denote the total connection cost when $j$ is also opened. If the maximum over unopened facilities of the ratio $\left(C-C_{j}-f_{j}\right) / f_{j}$ is positive, then open the facility that maximizes this ratio.

Finally, we describe the plane partitioning heuristic, which is not guaranteed to produce a solution within any constant factor. Figure 1 provides a visual reference.

\section{Trivial Heuristic}

(a) We partition the square into an $m \times m$ grid $\Gamma$ of subsquares $S_{p, q}, 1 \leq p, q \leq m$ of side length $\alpha$, and then open the facility $F_{p, q}$ closest to the center of each subsquare, assuming that there is one within distance $\alpha / \omega=\left(\frac{\log n}{n}\right)^{1 / 2}$ of its center.

(b) If any subsquare $S_{p, q}$ has no facility within distance $\alpha / \omega$ of its center, then open each $X_{i}$ in $S_{p, q}$ as a facility.

The Trivial Heuristic pays little attention to the structure of the instance, but, as we will prove in Section 3, it produces a solution which is asymptotically optimal whp. In fact, in 


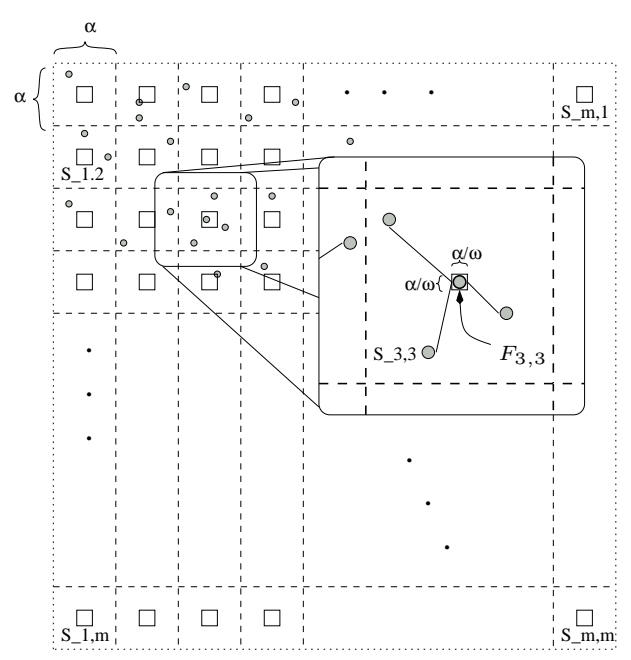

Figure 1: A schematic representation of the asymptotically optimal solution.

some sense, it is because it does not pay attention to the instance that it out-performs the approximation algorithms. All of the greedy algorithms are distracted by local deviations in city density, and (at least at first) they will open facilities at what amount to random points in the plane. This results in non-uniform coverage and requires some unlucky cities to suffer excessive connection costs.

\section{$3 \quad$ An asymptotically optimal solution}

In this section, we prove that the solution found by the Trivial Heuristic is asymptotically optimal. To do so, we obtain an upper bound on the cost of this solution and a matching lower bound on the dual of the LP-relaxation.

Let $H E U$ denote the total cost of the solution found by the Trivial Heuristic.

An intuition which explains the near optimality of this solution is that the cities and facilities are roughly uniformly distributed in the square, so the advantage of using the special structure of the instance is negligible.

To make this intuition rigorous, in the following 2 subsections, we obtain an upper bound on $H E U$ which holds whp, and a lower bound on $O P T$ which also holds whp and asymptotically matches the upper bound on $H E U$. But first we state and prove 2 lemmas that will aid in our analysis. 


\subsection{Some simple lemmas}

The following 2 lemmas will help us in analyzing the heuristic and the dual lower bound.

Lemma 1. Let $A_{1}, \ldots, A_{k}$ be subsets of $[0,1]^{2}$ each of area a, let $\mathcal{X}$ be a set of $n$ random points distributed uniformly and independently in $[0,1]^{2}$, and let $\lambda$ be a positive real with $\lambda \leq 1 / 3$. Then

$$
\begin{gathered}
\operatorname{Pr}\left[\exists i: A_{i} \cap \mathcal{X}=\emptyset\right] \leq k \cdot e^{-a n} \\
\operatorname{Pr}\left[\exists i:\left|A_{i} \cap \mathcal{X}\right| \notin(1 \pm \lambda) a n\right] \leq k \cdot 2 e^{-\lambda^{2} a n / 3}
\end{gathered}
$$

Proof (1) follows because the probability that a single point avoids $A_{i}$ is $1-a$ and $1-x \leq e^{-x}$ and the union bound.

(2) follows from Chernoff's bound and the union bound.

Lemma 2. Let $t$ be a positive real, let $F_{1}, \ldots, F_{k}$ be points in $[t, 1-t]^{2}$, let $\mathcal{X}$ be a set of $n$ random points distributed uniformly and independently in $[0,1]^{2}$, and let $\lambda$ be a positive real with $\lambda \leq 1 / 6$. For $i=1, \ldots, k$, let $Z_{i}=\sum_{\substack{X \in \mathcal{X} \\ d\left(X, F_{i}\right) \leq t}} d\left(X, F_{i}\right)$. Then

$$
\begin{aligned}
\mathbf{E}\left[Z_{i}\right] & =\frac{n(2 t)^{3}}{3} \\
\operatorname{Pr}\left[\exists i: Z_{i} \notin(1 \pm \lambda) \frac{n(2 t)^{3}}{3}\right] & \leq k \cdot 4 e^{-\lambda^{2}(2 t)^{2} n / 12} .
\end{aligned}
$$

\section{Proof}

We begin by considering the contribution of a particular point $X$ to $Z_{i}$. Conditioning on $d\left(X, F_{i}\right) \leq t$, the expected distance is

$$
\mathbf{E}\left[d\left(X, F_{i}\right) \mid d\left(X, F_{i}\right) \leq t\right]=t^{-2} \int_{0}^{t}(u \cdot 2 u) d u=\frac{2 t}{3} .
$$

We define $N_{i}$ to be the number of points within distance $t$ of $F_{i}$,

$$
N_{i}=\left|\left\{X \in \mathcal{X}: d\left(X, F_{i}\right) \leq t\right\}\right| .
$$

It follows from the linearity of expectations that

$$
\mathbf{E}\left[Z_{i} \mid N_{i}\right]=N_{i} \frac{2 t}{3}
$$

And, since $\mathbf{E}\left[N_{i}\right]=(2 t)^{2} n$, we have established (3),

$$
\mathbf{E}\left[Z_{i}\right]=\left((2 t)^{2} n\right) \frac{2 t}{3} .
$$


Conditioning on $N_{i}, Z_{i}$ is a sum of $N_{i}$ independent random variables in the range $[0, t]$. So Hoeffding's inequality gives

$$
\begin{aligned}
\operatorname{Pr}\left[Z_{i} \notin(1 \pm \lambda) N_{i} \frac{2 t}{3} \mid N_{i}\right] & \leq 2 e^{-2\left(\lambda N_{i} 2 t / 3\right)^{2} /\left(N_{i} t^{2}\right)} \\
& =2 e^{-8 \lambda^{2} N_{i} / 9} .
\end{aligned}
$$

Now, we apply Lemma 1 with $A_{i}=\left\{X: d\left(X, F_{i}\right) \leq t\right\}$ and (2) shows that the probability that some $N_{i}$ does not contain $(1 \pm \lambda)(2 t)^{2} n$ points is at most $k \cdot 2 e^{-\lambda^{2}(2 t)^{2} n / 3}$. Combining this with the conditional upper bound on the large deviation probability of $Z_{i}$ and the union bound gives

$$
\operatorname{Pr}\left[\exists i: Z_{i} \notin(1 \pm \lambda)\left((1 \pm \lambda)(2 t)^{2} n\right) \frac{2 t}{3}\right] \leq k \cdot 2 e^{-\lambda^{2}(2 t)^{2} n / 3}+k \cdot 2 e^{-8 \lambda^{2}(1-\lambda)(2 t)^{2} n / 9} .
$$

Since $\lambda \leq 1 / 3$, this simplifies to

$$
\operatorname{Pr}\left[\exists i: Z_{i} \notin(1 \pm \lambda)(2 t)^{3} n / 3\right] \leq 4 k e^{-\lambda^{2}(2 t)^{2} n / 3} .
$$

\section{$3.2 \quad$ An upper bound on $H E U$}

To achieve this goal, we define several events and random variables and bound probabilities related to them.

Let $\hat{F}_{p, q}$ be the point in the center of subsquare $S_{p, q}$.

We begin by showing that in each subsquare, there is likely to be a facility within distance $\alpha / \omega$ of $\hat{F}_{p, q}$ that we will open. To do this, we apply Lemma 1 with $k=m^{2}$ and $A_{p m+q}$ equal to the square within distance $\alpha / \omega$ of $\hat{F}_{p, q}$. Then, since area $\left(A_{p m+q}\right)=(2 \alpha / \omega)^{2}=\frac{4 \log n}{n},(1)$ shows that

$$
\operatorname{Pr}\left[\exists p, q: A_{p m+q} \cap \mathcal{X}=\emptyset\right] \leq m^{2} \cdot e^{-4 \log n}=o\left(n^{-3}\right) .
$$

Now we bound the transportation costs. We define a mapping $\pi$ so that for each $X_{i}$ with $X_{i} \in S_{p, q}$ and $F_{p, q}=X_{j}$ we have $\pi(i)=j$ to indicate that facility $j$ services city $i$. In the unlikely event that $A_{p m+q}$ is empty, we open all the facilities in $S_{p, q}$ and set $\pi(i)=i$ for each of them, which results in transportation cost 0 .

Note that, since $F_{p, q}$ is within $\alpha / \omega$ of $\hat{F}_{p, q}$, we have

$$
\sum_{X_{i} \in \mathcal{X}} d\left(X_{i}, X_{\pi(i)}\right) \leq \sum_{X_{i} \in \mathcal{X}} d\left(X_{i}, F_{p, q}\right)+n \alpha / \omega
$$


We apply Lemma 2 with $t=\alpha / 2, k=m^{2}, F_{p m+q}=\hat{F}_{p, q}$, and $\lambda=\omega^{-1}$. Then (3) and (6) together imply that

$$
\mathbf{E}\left[\sum_{X_{i} \in \mathcal{X}} d\left(X_{i}, X_{\pi}(i)\right)\right] \leq m^{2} \frac{n \alpha^{3}}{3}+n \alpha / \omega
$$

and (4) and (6) imply that

$$
\begin{aligned}
\operatorname{Pr}\left[\sum _ { X _ { i } \in \mathcal { X } } d \left(X_{i},\right.\right. & \left.\left.X_{\pi}(i)\right) \geq m^{2} \cdot\left(1+4 \omega^{-1}\right) \frac{n \alpha^{3}}{3}+\frac{n \alpha}{\omega}\right] \\
& \leq m^{2} \cdot 4 e^{-16 \omega^{-2} \alpha^{2} n / 12} \\
& =4 m^{2} e^{-4 \log n / 3} .
\end{aligned}
$$

Since there are $m^{2}$ facilities opened with probability at least $1-n^{-3}$, and there are at most $n$ facilities opened in even the most pathological point set, we may the bound expected total cost of the solution by

$$
\mathbf{E}[H E U]=\frac{n \alpha}{3}+n \alpha / \omega+m^{2} f+n f n^{-3}=\frac{1}{2} \alpha n(1+o(1)) .
$$

Finally, we observe that the probability that $H E U$ exceeds this bound tends to 0 ; the transportation cost is at most $\frac{n \alpha}{3}\left(1+O\left(\omega^{-1}\right)\right)$ with probability $1-o(1)$ and the probability that more than $m^{2}$ facilities open is $o(1)$. So we conclude that

$$
H E U \leq \frac{n \alpha}{2}(1+o(1)) \quad \text { whp. }
$$

\subsection{Lower bound on $O P T$}

To show this solution is asymptotically optimal, we will construct a solution to the dual of the strong LP relaxation (which has been studied since [3]):

\section{(LP-RELAX)}

$$
\begin{array}{rlrl}
\min & \sum_{j=1}^{n} f y_{j}+\sum_{i=1}^{n} \sum_{j=1}^{n} d\left(X_{i}, X_{j}\right) x_{i, j} \\
\text { subj. to } & \sum_{j=1}^{n} x_{i, j}=1 & 1 \leq i \leq n \\
0 \leq x_{i, j} \leq y_{j} & 1 \leq i, j \leq n .
\end{array}
$$

\section{(DUAL)}

$$
\begin{aligned}
& \max \quad \sum_{i=1}^{n} u_{i} \\
& \text { subj. to } \quad \sum_{i=1}^{n} v_{i, j} \leq f \quad 1 \leq j \leq n \\
& -v_{i, j}+u_{i} \leq d\left(X_{i}, X_{j}\right) \quad 1 \leq i, j \leq n \\
& v_{i, j} \geq 0 \quad 1 \leq i, j \leq n .
\end{aligned}
$$


We get a good solution to DUAL as follows:

$$
\begin{aligned}
u_{i} & = \begin{cases}\frac{\alpha}{2}\left(1-3 \omega^{-1}\right) & X_{i} \in[\alpha, 1-\alpha]^{2} . \\
0 & \text { otherwise. }\end{cases} \\
v_{i, j} & =\max \left\{u_{i}-d\left(X_{i}, X_{j}\right), 0\right\} .
\end{aligned}
$$

The fact that this solution is feasible whp follows from Lemma 1 and Lemma 2. We take $t=\frac{\alpha}{2}\left(1-3 \omega^{-1}\right), k=n, F_{i}=X_{i}$, and $\lambda=4 \omega^{-1}$. Then (4) shows that

$$
\begin{aligned}
\operatorname{Pr}\left[\exists i: Z_{i} \leq\left(1-4 \omega^{-1}\right) n(\alpha\right. & \left.\left.\left(1-3 \omega^{-1}\right)\right)^{3} / 3\right] \\
& \leq n \cdot 4 e^{-16 \omega^{-2}\left(\alpha\left(1-3 \omega^{-1}\right)\right)^{2} n / 12} \\
& =4 n e^{-16\left(1-3 \omega^{-1}\right)^{2} \log n / 12} \\
& =o(1) .
\end{aligned}
$$

Taking $A_{i}$ to be the $\alpha\left(1-3 \omega^{-1}\right) \times \alpha\left(1-3 \omega^{-1}\right)$ square centered at $X_{i},(2)$ shows that

$$
\begin{aligned}
\operatorname{Pr}\left[\exists i:\left|A_{i} \cap \mathcal{X}\right| \geq\left(1+4 \omega^{-1}\right)\right. & \left.\left(1-3 \omega^{-1}\right)^{2} \alpha^{2} n\right] \\
\leq & n \cdot 2 e^{-16 \omega^{-2}\left(1-3 \omega^{-1}\right)^{2} \alpha^{2} n / 3} \\
= & 2 n e^{-16\left(1-3 \omega^{-1}\right)^{2} \log n / 3} \\
= & o(1) .
\end{aligned}
$$

So whp for all $j$ we have

$$
\begin{aligned}
\sum_{i=1}^{n} v_{i, j} & =\sum_{X_{i} \in \mathcal{X}} \max \left\{\frac{\alpha}{2}\left(1-3 \omega^{-1}\right)-d\left(X_{i}, X_{j}\right), 0\right\} \\
& <\frac{n \alpha^{3}}{6}=f .
\end{aligned}
$$

Since the objective value of this solution asymptotically matches that of (7), we conclude that our "heuristic" is asymptotically optimal.

\section{Proof of Main Theorem}

To prove Theorem 1, in Section 4.1 we state and prove some lemmas which show that the structure of any near optimal solution must take a certain form. In particular, the solution must choose facilities to open so that, for most open facilities, the region of the plane which is closer to that facility than any other (the Voronoi cell) is approximately a square of a certain size and is approximately centered on the facility. Lemma 5 from Section 4.1 gives a 
quantitative version of this fact: it says roughly that if there are $\epsilon n$ facilities opened which violate the conditions then the solution will be a $1+\delta$ factor away from optimal.

To complete the proof of Theorem 1, in Section 4.2 we show that the approximation algorithms from Section 2 open too many facilities which do not meet the requirements given in Lemma 5 for a close to optimal solution whp.

\subsection{Properties of close-to-optimal solutions}

\subsubsection{Refining $\Gamma$ to super-grid $\Gamma_{1}$}

Now let $m_{1}=\left\lfloor\omega^{1 / 2}\right\rfloor m$ and let $\Gamma_{1}$ be the $m_{1} \times m_{1}$ super-grid of $\Gamma$ where each subsquare has side $\alpha_{1}=m_{1}^{-1}$. If we fix a subsquare $S$ of $\Gamma_{1}$ then the number of points $\nu_{S}$ of $\mathcal{X}$ which fall in $S$ is distributed as $\mathrm{B}\left(n, \alpha_{1}^{2}\right)$. Thus $\mathbf{E}\left(\nu_{S}\right)=\alpha_{1}^{2} n=\omega \log n(1+o(1))$. It follows from Lemma 1 , part (2) that

$$
\begin{aligned}
\operatorname{Pr}\left[\exists S \in \Gamma_{1}: \nu_{S} \notin(1\right. & \left.\left. \pm \omega^{-1 / 3}\right) \alpha_{1}^{2} n\right] \\
& \leq m_{1}^{2} \cdot 2 e^{-\omega^{-2 / 3} \alpha_{1}^{2} n / 3} \\
& <n \cdot 2 e^{-\omega^{1 / 3} \log n / 3}
\end{aligned}
$$

We use the term quite surely (qs) to describe a sequence of events which occurs with probability exceeding $1-O\left(n^{-k}\right)$ for any constant $k$. In this notation, we may say that

$$
\left|\nu_{S}-\alpha_{1}^{2} n\right| \leq \omega^{2 / 3} \log n, \forall S \in \Gamma_{1}, \quad \text { qs. }
$$

\subsubsection{An assignment which respects super-grid $\Gamma_{1}$}

For a set of facilities $\mathcal{F}$ and an assignment of cities to facilities $\phi: \mathcal{X} \rightarrow \mathcal{F}$ we let

$$
\kappa(\mathcal{F}, \phi)=f|\mathcal{F}|+\sum_{X \in \mathcal{X}} d(X, \phi(X)) .
$$

The assignment which maps points to their closest facility in $\mathcal{F}$ will be denoted $\phi_{\mathcal{F}}^{\star}$ so that

$$
c(\mathcal{F})=\kappa\left(\mathcal{F}, \phi_{\mathcal{F}}^{\star}\right)
$$

Consider a particular facility set $\mathcal{F}=\left\{F_{1}, F_{2}, \ldots, F_{k}\right\} \subseteq \mathcal{X}$. For each $F_{i}$ let $V_{i}$ be the Voronoi cell associated with $F_{i}$, which is to say $V_{i}$ is the set of points in $[0,1]^{2}$ which are at least as close (in $\ell_{\infty}$ norm) to $F_{i}$ as to any other member of $\mathcal{F}$.

We say an assignment $\phi$ respects $\Gamma_{1}$ if all the cities in a common subsquare of $\Gamma_{1}$ are assigned to the same facility by $\phi$.

The next lemma says that there is an assignment which respects $\Gamma_{1}$ and is not much worse than $\phi_{\mathcal{F}}^{\star}$. 
Lemma 3. There exists an assignment $\tilde{\phi}_{\mathcal{F}}$ that respects $\Gamma_{1}$ and has $\left|\kappa\left(\mathcal{F}, \tilde{\phi}_{\mathcal{F}}\right)\right|-\kappa\left(\mathcal{F}, \phi_{\mathcal{F}}^{\star}\right) \mid \leq$ $2 \alpha_{1} n$.

Proof The proof of the lemma is a shifting argument. For any assignment $\phi$, if there exists some $S \in \Gamma_{1}$ and $i \in[k]$ such that $V_{i} \cap S \cap \mathcal{X} \neq \emptyset$ and $S \backslash V_{i} \neq \emptyset$ then we make a slightly different assignment $\tilde{\phi}$ which assigns all cities in $S$ to the same facility. Let $i$ be the smallest index in $[k]$ such that cities in $S$ are assigned to $F_{i}$. Then we re-assign all $X_{j} \in S \backslash V_{i}$ to facility $F_{i}$. We claim that this adds at most $2 \alpha_{1}$ in transportation cost for each city. Indeed, suppose that $X_{j} \in S \cap V_{i^{\prime}}$ for $i^{\prime} \neq i$. Then $d\left(X_{j}, F_{i}\right) \leq d\left(X_{j}, X\right)+d\left(X, F_{i}\right)$. If $X \in V_{i} \cap S$, then we also have that $d\left(X, F_{i}\right) \leq d\left(X, X_{j}\right)+d\left(X_{j}, F_{i^{\prime}}\right)$, since $X$ is in $V_{i}$ and not $V_{j}$. So $d\left(X_{j}, F_{i}\right) \leq d\left(X_{j}, F_{i^{\prime}}\right)+2 d\left(X, X_{j}\right)$. Since $X$ and $X_{j}$ are both in $S, d\left(X, X_{j}\right) \leq \alpha_{1}$.

By starting with $\phi_{\mathcal{F}}^{\star}$ and repeating this shifting we eventually arrive with an assignment $\tilde{\phi}_{\mathcal{F}}$ (since assignments to cities in each cell are adjusted at most once). This assignment respects $\Gamma_{1}$ by construction, and (again because each city is reassigned at most once) we have

$$
\kappa\left(\mathcal{F}, \tilde{\phi}_{\mathcal{F}}\right) \leq \kappa\left(\mathcal{F}, \phi_{\mathcal{F}}^{\star}\right)+2 \alpha_{1} n
$$

\subsubsection{The likely cost per facility under $\tilde{\phi}_{\mathcal{F}}$}

For $F_{i} \in \mathcal{F}$, let the $\tilde{V}_{i}$ be the union of the subsquares in $\Gamma_{1}$ which contain cities which are mapped to $F_{i}$ by $\tilde{\phi}_{\mathcal{F}}$ (we think of $\tilde{V}_{i}$ as the "quantized Voronoi cell" of $F_{i}$ ). Let $\eta_{i}$ denote the number of subsquares in $\tilde{V}_{i}$. Let $\mathcal{X}_{i}=\mathcal{X} \cap \tilde{V}_{i}$ and let

$$
c_{i}=\sum_{X \in \mathcal{X}_{i}} d\left(X, F_{i}\right)
$$

Note that, because of the way $\tilde{\phi}_{\mathcal{F}}$ was constructed, for any $\Gamma_{1}$-subsquare $S$, if $S \subseteq V_{i}$ then $S \subseteq \tilde{V}_{i}$

We say that $\tilde{V}_{i}$ is an $\epsilon$-quasi-square if there exists a square $S$ centered at $F_{i}$ such that $\max \left\{\operatorname{area}\left(S \backslash \tilde{V}_{i}\right), \operatorname{area}\left(\tilde{V}_{i} \backslash S\right)\right\} \leq \epsilon \operatorname{area}\left(\tilde{V}_{i}\right)$.

Lemma 4. Assume that (8) holds. Assume that $\epsilon \gg \alpha_{1}$. Then whpthe following hold for all $i$

(i) $c_{i} \geq \frac{1}{3} n\left(1-\omega^{-1 / 3}\right) \operatorname{area}\left(\tilde{V}_{i}\right)^{3 / 2}$.

(ii) If $\tilde{V}_{i}$ is not an $\epsilon$-quasi-square then $c_{i} \geq \frac{1+\epsilon^{2} / 4}{3} n\left(1-\omega^{-1 / 3}\right) \operatorname{area}\left(\tilde{V}_{i}\right)^{3 / 2}$. 
Proof In light of (8), this lemma reduces to a pair of geometric facts about collections of squares. However, it is convenient for us to prove the facts via linear programming.

We begin by establishing part (i) of the lemma. Fix $i$. For every $j$ define $U_{j}=\left\{S \in \Gamma_{1}\right.$ : $S \subseteq \tilde{V}_{i}$ and $\left.j \alpha_{1} \leq d\left(S, F_{i}\right)<(j+1) \alpha_{1}\right\}$. We have $\left|U_{j}\right| \leq 8 j+4$. Let $k$ be such that $U_{j}=\emptyset$ for every $j>k$. Such $k$ exists because $\tilde{V}_{i}$ is compact. By counting the number of $\Gamma_{1}$-squares in $\tilde{V}_{i}$ we get

$$
\sum_{j=0}^{k}\left|U_{j}\right|=\eta_{i}=\operatorname{area}\left(\tilde{V}_{i}\right) / \alpha_{1}^{2} .
$$

Now,

$$
\begin{aligned}
c_{i} & =\sum_{X \in \mathcal{X}_{i}} d\left(X, F_{i}\right) \\
& =\sum_{S \in \Gamma_{1}: S \subseteq \tilde{V}_{i}} \sum_{X \in S} d\left(X, F_{i}\right) \\
& \geq \sum_{S \subseteq \tilde{V}_{i}} \nu_{S} d\left(F_{i}, S\right) \\
& \geq\left(\alpha_{1}^{2} n-\omega^{2 / 3} \log n\right) \sum_{S \subseteq \tilde{V}_{i}} d\left(F_{i}, S\right) \\
& =\left(\alpha_{1}^{2} n-\omega^{2 / 3} \log n\right) \sum_{j=0}^{k} \sum_{S \in U_{j}} d\left(F_{i}, S\right) \\
& \geq\left(\alpha_{1}^{2} n-\omega^{2 / 3} \log n\right) \alpha_{1} \sum_{j=0}^{k} j\left|U_{j}\right|
\end{aligned}
$$

As we want a lower bound for $c_{i}$ we consider the primal-dual pair

(P.i)

$$
\begin{aligned}
& \min \quad \sum_{j=0}^{k} j x_{j} \\
& \text { subj to } \quad x_{j} \leq 8 j+4 j=0,1, \ldots, k \\
& \sum_{j=0}^{k} x_{j}=\eta_{i} \\
& x_{j} \geq 0 \quad j=0,1, \ldots, k \\
& \text { (D.i) } \\
& \max \quad \eta_{i} z-\sum_{j=0}^{k}(8 j+4) y_{j} \\
& \text { subj. to } z-y_{j} \leq j \quad j=0,1, \ldots, k \\
& y_{j} \geq 0 \quad j=0,1, \ldots, k
\end{aligned}
$$


A feasible solution for D.i is to take $z=\eta_{i}^{1 / 2} / 2$ and $y_{j}=\max \left(\eta_{i}^{1 / 2} / 2-j, 0\right), j=0, \ldots, k$ with dual value $\geq \eta_{i}^{3 / 2} / 3$, and then $\sum_{j=0}^{k} j\left|U_{j}\right| \geq \eta_{i}^{3 / 2} / 3=\operatorname{area}\left(V_{i}\right)^{3 / 2} / 3 \alpha_{1}^{3}$.

(The expression $\sum_{j=0}^{\ell}(8 j+4)(A-j)=4 A(\ell+1)^{2}-\left(\frac{8}{3} \ell^{3}+6 \ell^{2}+\frac{4}{3} \ell\right)$ will no doubt help the reader to verify the above claim.)

Now we show that part (ii) of the lemma holds. We introduce extra constraints in the linear program above in order to enforce the condition that $\tilde{V}_{i}$ is not an $\epsilon$-quasi-square. For this, assume that $\tilde{V}_{i}$ is not an $\epsilon$-quasi-square, let $\ell=\left\lfloor\eta_{i}^{1 / 2} / 2\right\rfloor$ and let $S$ be the square of side $2 \ell \alpha_{1}$ centered a $F_{i}$. Then area $\left(\tilde{V}_{i}\right) \geq \operatorname{area}(S) \geq(1-\epsilon)$ area $\left(\tilde{V}_{i}\right)$ and therefore area $(S \cap$ $\left.\tilde{V}_{i}\right)<(1-\epsilon)$ area $\left(\tilde{V}_{i}\right)$, otherwise area $\left(\tilde{V}_{i} \backslash S\right)=\operatorname{area}\left(\tilde{V}_{i}\right)-\operatorname{area}\left(\tilde{V}_{i} \cap S\right) \leq \epsilon \operatorname{area}\left(\tilde{V}_{i}\right)$ and $\operatorname{area}\left(S \backslash \tilde{V}_{i}\right)=\operatorname{area}(S)-\operatorname{area}\left(S \cap \tilde{V}_{i}\right) \leq \epsilon \operatorname{area}\left(\tilde{V}_{i}\right)$. Then $\sum_{j=0}^{\ell}\left|U_{j}\right|=\operatorname{area}\left(S \cap \tilde{V}_{1}\right) / \alpha_{1}^{2} \leq$ $(1-\epsilon)$ area $\left(\tilde{V}_{i}\right) / \alpha_{1}^{2}=(1-\epsilon) \eta_{i}$, so we consider the primal-dual pair

(P.ii)

$$
\min \quad \begin{aligned}
& \sum_{j=0}^{k} j x_{j} \\
& \text { subj to } x_{j} \leq 8 j+4 \quad j=0,1, \ldots, k \\
& \sum_{j=0}^{k} x_{j}=\eta_{i} \\
& \sum_{j=0}^{\ell} x_{j} \leq(1-\epsilon) \eta_{i} \\
& x_{j} \geq 0 \quad j=0,1, \ldots, k
\end{aligned}
$$

(D.ii)

$$
\begin{aligned}
& \max \quad \eta_{i} z-(1-\epsilon) \eta_{i} z_{1}-\sum_{j=0}^{k}(8 j+4) y_{j} \\
& \text { subj to } z-z_{1}-y_{j} \leq j \quad j=0,1, \ldots, \ell \\
& z-y_{j} \leq j \quad j=\ell+1, \ldots, k \\
& z_{1} \geq 0 \\
& y_{j} \geq 0 \quad j=0,1, \ldots, k
\end{aligned}
$$

A feasible solution for D.ii is $z=(1+\epsilon) \eta_{i}^{1 / 2} / 2, z_{1}=\epsilon \eta_{i}^{1 / 2} / 2, y_{j}=(1-\epsilon / 2) \eta_{i}^{1 / 2} / 2-j$, $j=0, \ldots, \ell$ and $y_{j}=\max \left((1+\epsilon / 2) \eta_{i}^{1 / 2} / 2-j, 0\right), j=\ell+1, \ldots, k$ with dual value $\geq$ $\left(1+\epsilon^{2} / 4\right) \eta_{i}^{3 / 2} / 3$, and then $\sum_{j=0}^{k} j\left|U_{j}\right| \geq\left(1+\epsilon^{2} / 4\right) \eta_{i}^{3 / 2} / 3 \geq\left(1+\epsilon^{2} / 4\right)$ area $\left(V_{i}\right)^{3 / 2} / 3 \alpha_{1}^{3}$.

\subsubsection{The structure of any near optimal solution}

We continue by proving a property of any near optimal solution to the UFLP.

Lemma 5. Assume that (8) holds. Let $\epsilon$ be a sufficiently small constant, and let $\mathcal{F} \subseteq \mathcal{X}$ with $\kappa\left(\mathcal{F}, \phi_{\mathcal{F}}^{\star}\right) \leq(1+\epsilon) \alpha n / 2$. Then for $\epsilon_{1}=5 \epsilon^{1 / 2}$, 
(a) $|\mathcal{F}| \in\left[\left(1-\epsilon_{1}\right) m^{2},\left(1+\epsilon_{1}\right) m^{2}\right]$.

(b) Suppose that $\theta_{1}=2 \epsilon^{1 / 3}$ and $\theta_{2}=4 \epsilon^{1 / 3}$ and $\epsilon_{0}=3 \epsilon^{1 / 3}$. Then at least $\left(1-2 \theta_{2}\right) m^{2}$ of the points $F_{i} \in \mathcal{F}$ are such that $\tilde{V}_{i}$ is an $\epsilon_{0}$-quasi-square of area in the range $[(1-$ $\left.\theta_{1}\right) \alpha^{2},\left(1+\theta_{1}\right) \alpha^{2}$.

Proof Let $\mathcal{F}=\left\{F_{1}, F_{2}, \ldots, F_{k}\right\}$ and let $a_{i}=\left|\tilde{V}_{i}\right|$ for $1 \leq i \leq k$. Let $J=\{j$ : $\tilde{V}_{j}$ is not a $\epsilon_{0}$-quasi-square $\}$. Applying Lemma 4 and equation (9) we see that

$$
\kappa\left(\mathcal{F}, \phi_{\mathcal{F}}^{\star}\right) \geq k f+\frac{1-\omega^{-1 / 3}}{3} n\left(\sum_{i=1}^{k} a_{i}^{3 / 2}+\frac{\epsilon_{0}^{2}}{12} \sum_{j \in J} a_{j}^{3 / 2}\right)-2 \alpha_{1} n .
$$

Now let $a_{j}=\frac{1+x_{j}}{k}$, where $-1 \leq x_{j}$ and $\sum_{j=1}^{k} x_{j}=0$.

By examining the power series for $(1+x)^{3 / 2}$ when $|x| \leq 1$ and using elementary calculus for $x>1$ we see that

$$
(1+x)^{3 / 2} \geq 1+\frac{3}{2} x+\min \left\{1, \frac{1}{4} x^{2}\right\} \quad x \geq-1 .
$$

It follows from (10) that

$$
\begin{aligned}
& \kappa\left(\mathcal{F}, \phi_{\mathcal{F}}^{\star}\right) \geq \\
& k f+\left(\frac{1-\omega^{-1 / 3}}{3} n\right) \times\left(k^{-1 / 2}+k^{-3 / 2} \sum_{i=1}^{k} \min \left\{1, \frac{1}{4} x_{i}^{2}\right\}+\frac{\epsilon_{0}^{2}}{12} \sum_{j \in J} a_{j}^{3 / 2}\right)-2 \alpha_{1} n
\end{aligned}
$$

Now, let $k=(1+\theta) \alpha^{-2}$ for some $\theta \geq-1$ and assume wlog that $|\theta| \gg \omega^{-1 / 6}$. Notice that from (10) that we can assume $\theta<3$, otherwise $k f \geq \frac{4}{6} \alpha$. If $\theta \in[-1,3]$ then $\frac{1}{(1+\theta)^{1 / 2}} \geq$ $1-\frac{1}{2} \theta+\frac{1}{16} \theta^{2}$, and we get

$$
\begin{aligned}
k f+\frac{1-\omega^{-1 / 3}}{3} n k^{-1 / 2}-2 \alpha_{1} n \geq & \\
\frac{(1+\theta)}{6} \alpha n+\frac{\left(1-\omega^{-1 / 3}\right)}{3} \alpha n\left(1-\frac{1}{2} \theta+\frac{1}{16} \theta^{2}\right)-2 \alpha_{1} n \geq & \frac{\alpha n}{2}\left(1+\frac{\theta^{2}}{25}\right) .
\end{aligned}
$$

And using (12) we get

$$
\kappa\left(\mathcal{F}, \phi_{\mathcal{F}}^{\star}\right) \geq \frac{\alpha n}{2}\left(1+\frac{\theta^{2}}{25}\right)+\frac{n}{4}\left(k^{-3 / 2} \sum_{i=1}^{k} \min \left\{1, \frac{1}{4} x_{i}^{2}\right\}+\frac{\epsilon_{0}^{2}}{12} \sum_{j \in J} a_{j}^{3 / 2}\right) .
$$


Part (a) follows from (14): $(1+\epsilon) \alpha n / 2 \geq \kappa\left(\mathcal{F}, \phi_{\mathcal{F}}^{\star}\right) \geq \frac{\alpha n}{2}\left(1+\frac{\theta^{2}}{25}\right)$ and so $|\theta| \leq \epsilon^{1 / 2} / 5$.

Using (14) again we get

$$
\kappa\left(\mathcal{F}, \phi_{\mathcal{F}}^{\star}\right) \geq \frac{1}{2} \alpha n+\frac{n}{4 k^{3 / 2}} \sum_{j=1}^{k} \min \left\{1, \frac{1}{4} x_{j}^{2}\right\},
$$

So if $B=\left\{j:\left|x_{j}\right| \geq \theta_{1}\right\}$ and $|B| \geq \beta k$ for $\theta_{1}, \beta \leq 1$, we have $\kappa\left(\mathcal{F}, \phi_{\mathcal{F}}^{\star}\right) \geq \frac{1}{2} \alpha n+\frac{\theta_{1}^{2} \beta}{16\left(1+\epsilon_{1}\right)^{1 / 2}} \alpha n$. Setting $\theta_{1}=2 \epsilon^{1 / 3}$ we get $\beta \leq \theta_{2}=4 \epsilon^{1 / 3}$. Returning once again to (14) we write

$$
\begin{aligned}
\kappa\left(\mathcal{F}, \phi_{\mathcal{F}}^{\star}\right) & \geq \frac{1}{2} \alpha n+\frac{\epsilon_{0}^{2}}{48} n \sum_{j \in J} a_{j}^{3 / 2} \\
& \geq \frac{1}{2} \alpha n+\frac{3 \epsilon^{2 / 3}}{16} n\left(|J|-\theta_{2} k\right)\left(\frac{1-\theta_{1}}{k}\right)^{3 / 2} .
\end{aligned}
$$

Thus, if $|J| \geq 2 \theta_{2} m^{2}$ then

$$
\begin{aligned}
\kappa\left(\mathcal{F}, \phi_{\mathcal{F}}^{\star}\right) & \geq \frac{1}{2} \alpha n+\frac{3 \epsilon^{2 / 3}}{16} n \theta_{2}\left(2 m^{2}-k\right)\left(\frac{1-\theta_{1}}{k}\right)^{3 / 2} \\
& \geq \frac{1}{2} \alpha n+\frac{12}{16} \epsilon n\left(1-\epsilon_{1}\right) m^{2}\left(\frac{1-2 \epsilon^{1 / 3}}{\left(1+\epsilon_{1}\right) m^{2}}\right)^{3 / 2} \\
& \geq \frac{1}{2} \alpha n+\frac{11}{16} \epsilon \alpha n .
\end{aligned}
$$

\subsection{Properties of Solutions Found by Greedy Approximation Al- gorithms}

The goal of this section is to use the characterization of close-to-optimal solutions obtained in Section 4.1 to show that the greedy approximation algorithms described in Section 2 find solutions which are not asymptotically optimal. This is achieved by considering the behavior of the algorithm on a $14 \alpha \times 7 \alpha$ rectangular subregion of the unit square, and showing that, with constant probability, this region contains a facility for which the Vornoi region is not $\epsilon$-quasi-square.

The intuition which motivates this approach is this: the candidate facilities which open in the subregion will do so at random locations, thus there is no reason to expect these random locations to result in nice Vornoi cells. Making this intuitive explaination rigorous requires some work because of the complicated dependencies between which facilities are opened. For example, all the approximation algorithms track a level of "funding" available 
for opening a candidate facility (in Approximation Algorithm 1, the funds for city $j$ are $\sum_{i \in U} \max \left\{0, \delta_{i}-d(i, j)\right\}$, and in Approximation Algorithm 2 and 3, the funds are at least this much.) The funds available to a facility at time $t$ is a difficult random variable to deal with, and we must work around this difficulty.

Let $p f(X, t)$ denote the potential funds at point $X$ at time $t$, given by

$$
p f(X, t)=\sum_{i \in C} \max \left\{0, t-d\left(X_{i}, X\right)\right\} .
$$

This is the level of funding available to open a facility at $X$ if no other facilities have already opened within distance $2 t$ of $X$.

Let $T(X)=\min \{\min \{t: p f(X, t)=f\}, \alpha\}$ be the earliest opening time of point $X$ (which is truncated at time $\alpha$, because we want $T(X)$ to only depend on the position of nearby points).

We note that $\mathbf{E}(p f(X, \alpha))=f$ and $p f(X, \alpha)$ is the sum of $n$ independent bounded random variables and so the Central Limit Theorem implies that

$$
\operatorname{Pr}(p f(X, \alpha) \geq f)=\frac{1}{2}-o(1) .
$$

Consider concentric squares, $S_{1}, S_{2}, \ldots$, where $S_{i}$ is an $i \alpha \times i \alpha$ square (see Figure 2 for visual reference). Some facility $X^{\star}$ in $S_{5}$ has the minimum value of $T(X)$ among all facilities in $S_{5}$, and which one it is only depends on the configuration of points in $S_{7}$.

Note that if $X^{\star}$ is in $S_{1}$, (and $T\left(X^{\star}\right)<\alpha$ ) then (in all 3 of the greedy approximation algorithms) $X^{\star}$ actually opens at time $T\left(X^{\star}\right)$, because no cities within distance $\alpha$ of $S_{1}$ are connected (because no facilities within $2 \alpha$ of $S_{1}$ are open; in other words, no facilities besides $X^{\star}$ are open in $S_{5}$.) Since nothing within $\alpha$ of $X^{\star}$ is connected,

$$
\sum_{i \in U} \max \left\{0, \delta_{i}-d\left(X_{i}, X^{\star}\right)\right\}=\sum_{i \in C} \max \left\{0, T\left(X^{\star}\right)-d\left(X_{i}, X^{\star}\right)\right\}
$$

We will partition $S_{7}$ into subsquares of size $\alpha / 4$, and obtain a constant lower bound on the probability $X^{\star}$ appears in one of these subsquares that is contained in $S_{1}$. For $(p, q) \in[4]^{2}$, let $Q_{p, q}$, denote such a subsquare (with side length $\alpha / 4$ that is contained in $S_{1}$ ). Figure 2 provides a visual reference.

Lemma 6. Let $X^{\star}$ be the facility in $S_{5}$ which minimizes $T(X)$ over $X \in \mathcal{X} \cap S_{5}$. There exists an absolute constant $\gamma_{0}$ such that for any $(p, q) \in[4]^{2}$,

$$
\operatorname{Pr}\left[X^{\star} \in Q_{p, q} \text { and } p f\left(X^{\star}, \alpha\right) \geq f\right] \geq \gamma_{0} .
$$




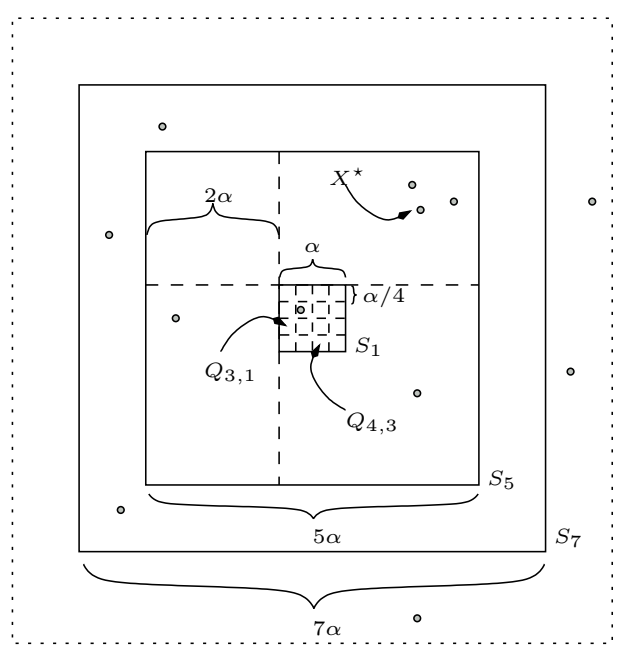

Figure 2: Concentric squares $S_{1}, S_{5}$, and $S_{7}$.

Proof We write $\mathcal{A}_{p, q}^{\star}=\left\{X^{\star} \in Q_{p, q}\right\}$ and $\mathcal{B}^{\star}=\left\{p f\left(X^{\star}, \alpha\right) \geq f\right\}$. We then consider the analogous question for $\mathcal{X}^{\prime}=\mathcal{X} \cap S_{7}$, where the edges of $S_{7}$ have been identified to "wrap-around", making the distance function

$d_{W}\left(\left(x_{1}, y_{1}\right),\left(x_{2}, y_{2}\right)\right)=\max \left\{\min \left\{\left|x_{1}-x_{2}\right|, 7 \alpha-\left|x_{1}-x_{2}\right|\right\}, \min \left\{\left|y_{1}-y_{2}\right|, 7 \alpha-\left|y_{1}-y_{2}\right|\right\}\right\}$.

(This makes the space a torus topologically.) In this case, some $\alpha / 4 \times \alpha / 4$ subsquare of $S_{7}$ contains the facility $X^{\star}$ which minimizes $T(X)$ and, by symmetry, each subsquare is equally likely to contain it. So the probability that $X^{\star}$ is in $Q_{p, q}$ is the same as the probability that it is in any of the $(7 \cdot 4)^{2}$ subsquares, which is exactly $1 /(7 \cdot 4)^{2}$. Using $\mathbf{P r}^{\prime}$ for this model and double $\star$ 's to distinguish the $S_{7}$ case from the $S_{5}$ case, we have

$$
\operatorname{Pr}^{\prime}\left(\mathcal{B}^{\star \star}\right)=\sum_{(a, b) \in[7 \cdot 4]^{2}} \operatorname{Pr}^{\prime}\left(\mathcal{B}^{\star} \wedge \mathcal{A}_{a, b}^{\star \star}\right)=(7 \cdot 4)^{2} \operatorname{Pr}^{\prime}\left(\mathcal{B}^{\star \star} \wedge \mathcal{A}_{p, q}^{\star \star}\right),
$$

giving

$$
\operatorname{Pr}^{\prime}\left(\mathcal{B}^{\star \star} \wedge \mathcal{A}_{p, q}^{\star \star}\right) \geq \frac{1}{3(7 \cdot 4)^{2}}
$$

Now, we remove the wrap-around on $S_{7}$ but continue to ignore all the points of $\mathcal{X}$ that lie outside $S_{7}$ (i.e. consider the potential funds for point set $\mathcal{X}^{\prime}=\mathcal{X} \cap S_{7}$ under the $\ell_{\infty}$ distance). This change can only affect $T(X)$ for a point $X$ which lies within distance $\alpha$ of the boundary of $S_{7}$, and for such an $X$, the change can only makes $T(X)$ larger than it was in the case with wrap-around. So every $\mathcal{X}$ which yielded $\mathcal{B}^{\star \star}$ and $X^{\star \star}$ in $Q_{p, q}$ with the wrap-around distance will have $\mathcal{B}^{\star}$ and the same $X^{\star}$ in this case. So the probability that $\mathcal{B}^{\star}$ occurs and $Q_{p, q}$ contains the point which opens first when $\mathcal{X}^{\prime}=\mathcal{X} \cap S_{7}$ is at least $(7 \cdot 4)^{-2} / 3$.

Finally, we return to the original set $\mathcal{X}$, and note that considering the contributions of points outside of $S_{7}$ to the potential funds does not affect $T(X)$ for any $X$ in $S_{5}$. So the 


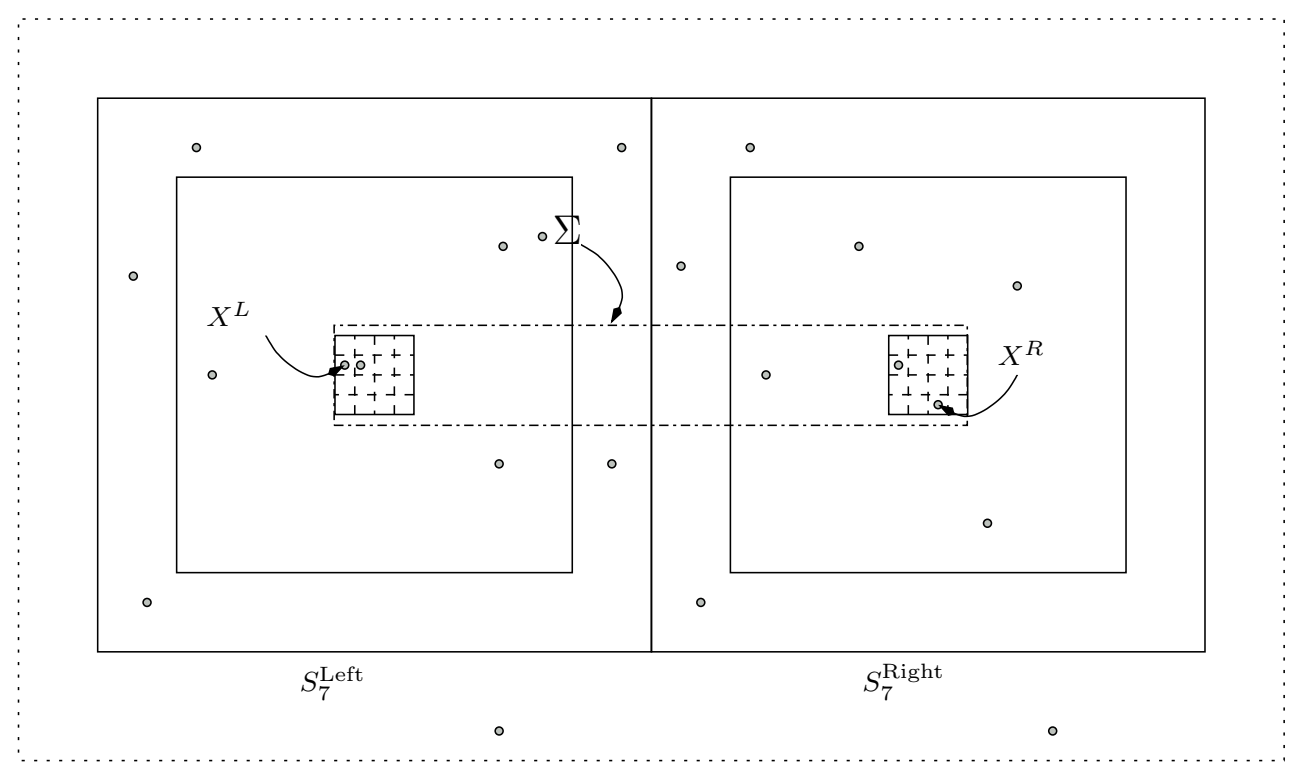

Figure 3: Two side-by-side copies of $S_{7}$

probability that $Q_{p, q}$ contains the point which opens first in $S_{5}$ with respect to $\mathcal{X}$ is at least the probability that $Q_{p, q}$ contains the point which opens first in $S_{7}$ with respect to $\mathcal{X}^{\prime}=\mathcal{X} \cap S_{7}$. The previous paragraph showed that this is at least $(7 \cdot 4)^{-2} / 3$.

Now consider 2 side-by-side copies of $S_{7}$, as shown in Figure 3 . Let $\mathcal{B}_{1}$ be the event that, in the left copy of $S_{7}$, the facility $X^{L}$ which minimizes $T(X)$ in $S_{5}^{L}$ is in $Q_{q, 1}^{L}$ for some $q$. Let $\mathcal{B}_{2}$ be the event that, in the right copy of $S_{7}$, the facility $X^{R}$ which minimizes $T(X)$ in $S_{5}^{R}$ is in $Q_{q^{\prime}, 3}^{R}$ for some $q^{\prime}$. Because $Q_{q, 1}^{L}$ and $Q_{q^{\prime}, 3}^{R}$ are sufficiently far apart, $\operatorname{Pr}\left[\mathcal{B}_{1} \mid \mathcal{B}_{2}\right]=\operatorname{Pr}\left[\mathcal{B}_{1}\right]$, and so $\operatorname{Pr}\left[\mathcal{B}_{1} \mathcal{B}_{2}\right] \geq \gamma_{0}^{2}$.

Suppose now that $\mathcal{B}_{1}$ and $\mathcal{B}_{2}$ occur. Let $\Sigma$ be the $(1+\epsilon) \alpha \times 8 \alpha$ strip containing $S_{1}^{L}$ and $S_{1}^{R}$ (so that $S_{1}^{L}, S_{1}^{R}$ are located symmetrically at distance $\epsilon \alpha / 2$ from the horizontal borders of $\Sigma$, as depicted in Figure 3.) Here $\epsilon$ is some sufficiently small positive constant. Let $I$ be the index set of those open facilities whose quantized Voronoi cells $\tilde{V}_{i}$ meet the strip $\Sigma$.

Lemma 7. Whp there must be some facility $i \in I$ for which $\tilde{V}_{i}$ is not $\epsilon^{3}$-quasi-square with area in $\left(1 \pm \epsilon^{3}\right) \alpha^{2}$.

Proof Assume for the sake of contradiction that this is not the case. Each such Voronoi region $\tilde{V}_{i}$ can therefore be associated with a square $W_{i}$ of side in the range $\left(1 \pm \epsilon^{3}\right) \alpha$. Furthermore, any two such squares have a common area of at most $\epsilon^{3} \alpha^{2}$. Whp there is no open facility $j$ at distance $2 \alpha$ or more from $\Sigma$ for which the quantized Voronoi region $\tilde{V}_{j}$ intersects $\Sigma$ (every point in $\mathcal{X} \cap \Sigma$ is connected to a closer open facility). Thus $|I| \leq \frac{8(5+\epsilon)}{1-\epsilon / 2}<50$. It follows that all but an area of at most $50 \epsilon^{3} \alpha^{2}$ of $\Sigma$ is covered by the $W_{i}$, for $i \in I$. Now let $\Sigma_{1}$ denote a strip of length $8 \alpha$ and thickness $\epsilon \alpha / 4$ running across the middle of $\Sigma$. Any 
sub-strip of $\Sigma_{1}$ which is of length $\epsilon \alpha$ is of area $\epsilon^{2} \alpha^{2} / 4$ and so will contain members of $\mathcal{X}$ which are covered by some $W_{i}, i \in I$.

If the center of this $W_{i}$ is outside $\Sigma$ then $W_{i}$ has side at least $(1+\epsilon / 4) \alpha$, which contradicts our assumption. So let $J$ be the set of facilities $j$ with center in $\Sigma$ for which there is a member of $\Sigma_{1}$ contained in $W_{j}$. If any of these facilities is not $\epsilon^{3}$-quasi-square then we are done, so we may assume that they all are. There is an open facility in $S_{q, 1}^{L}$ and in $S_{q^{\prime}, 3}^{R}$, and these facilities cover squares of side at least $\alpha$. Thus the other members of $J$ appear in a substrip with length between $7.25 \alpha$ and $7.75 \alpha$. If there are 6 or fewer open facilities in this the strip bounding the 2 copies, then some pair of facilities are at least $1.04 \alpha$ apart. Therefore, one of them, call it $F_{i}$ has a $W_{i}$ with side at least $\left(1.04-100 \epsilon^{3}\right) \alpha$, contradiction. On the other hand, if there are 7 or more facilities in the strip, then some pair are at most .96 $\alpha$ apart, and so some $F_{i}$ has a $W_{i}$ with side at most $\left(.96+\frac{2 \epsilon^{3}}{.48}\right) \alpha$, contradiction.

Since the event $\mathcal{B}_{1} \mathcal{B}_{2}$ occurs independently in sufficiently separated disjoint regions of the square (modulo there being enough points in the cell), whp we will have $\Omega\left(m^{2}\right)$ facilities for which $\tilde{V}_{i}$ is not an $\epsilon^{3}$-quasi-square with area $\left(1 \pm \epsilon^{3}\right) \alpha$. So Lemma 5 finishes the proof of the Theorem.

\section{References}

[1] S. Ahn, C. Cooper, G. Cornuéjols and A. M. Frieze, Probabilistic analysis of a relaxation for the $k$-median problem, Mathematics of Operations Research 13 (1988) 1-31.

[2] F. Barahona and F. A. Chudak, Solving large scale uncapacitated facility location problems, in Approximation and Complexity in Numerical Optimization: Continuous and Discrete Problems, Kluwer Academic Publishers, (1999).

[3] M. L. Balinski, Integer Programming: Methods, uses, computation, Management Science, 12(3):253-313, 1965.

[4] M. Charikar and S. Guha, Improved combinatorial algorithms for facility location and $k$-median problems, Proc. of the 40th Annual IEEE Symposium on Foundations of Computer Science, (1999), 378-388.

[5] F. A. Chudak and D. B. Shmoys, Improved approximation algorithms for the uncapacitated facility location problem, to appear in SIAM Journal of Computing.

[6] E. G. Coffman Jr., C. Courcoubetis, M. R. Garey, D. S. Johnson, P. W. Shor, R. R. Weber, and M. Yannakakis. Perfect packing theorems and the average-case behaviour of optimal and online bin packing. SIAM Review, 44(1):95-108, 2002.

[7] A. Coja-Oghlan, S. O. Krumke, and T. Nierhoff, A Heuristic for the Stacker Crane Problem on Trees Which Is Almost Surely Exact, Lecture Notes in Computer Science, Volume 2906, Jan 2003, 605-614 
[8] G. Cornuéjols, G. L. Nemhauser and L. A. Wolsey, The Uncapacitated Facility Location Problem, Discrete Location Theory, (1990).

[9] U. Feige, and J. Kilian, Heuristics for semirandom graph problems, J. Comput. Syst. Sci. 63, 4 (Dec. 2001), 639-673.

[10] M. Hoefer. Experimental comparison of heuristic and approximation algorithms for uncapacitated facility location. Proc. 2nd Intl. Workshop on Experimental and Efficient Algorithms (WEA 2003), LNCS 2647, (2003) 165-178.

[11] K. Jain, M. Mahdian, E. Markakis, A. Saberi, and V. V. Vazirani, Greedy facility location algorithms analyzed using dual fitting with factor-revealing LP, Journal of the ACM, 50 (6), (2003) 795-824.

[12] K. Jain and V. V. Vazirani, Primal-dual approximation algorithms for metric facility location and $k$-median problems, Proc. of the 40th Annual IEEE Symposium on Foundations of Computer Science (1999) 2-13.

[13] J. Krarup and P. M. Pruzan, The simple plant location problem: Survey and synthesis, European Journal of Operational Research 12 (1983) 36-81.

[14] M. R. Korupolu, C. G. Plaxton, and R. Rajaraman, Analysis of a local search heuristic for facility location problems, Proc. of the 9th Annual ACM-SIAM symposium on discrete algorithms (1998) 1-10.

[15] M. Mahdian, Y. Ye, and J. Zhang, Improved Approximation Algorithms for Metric Facility Location Problems, Proc. of the 5th Intl. Workshop on Approximation Algorithms for Combinatorial Optimization (2002) 229-242.

[16] M. Penrose, Random Geometric Graphs, Oxford University Press, (2003).

[17] D. B. Shmoys, E. Tardos, and K. I. Aardal, Approximation algorithms for facility location problems, Proc. of the 29th Annual ACM Symposium on the Theory of Computation (1997) 265-274.

[18] J. M. Steele, Probability Theory and Combinatorial Optimization CBMS-NSF Regional Conference Series in Applied Mathematics - Volume 69, SIAM, (1997).

[19] M. Sviridenko, An Improved Approximation Algorithm for the Metric Uncapacitated Facility Location Problem, Proc. of the Conference on Integer Programming and Combinatorial Optimization (IPCO02), LNCS 2337, (2002) 230-239.

[20] J. E. Yukich, Probability Theory of Classical Euclidean Optimization Problems, SpringerVerlag, (1998). 